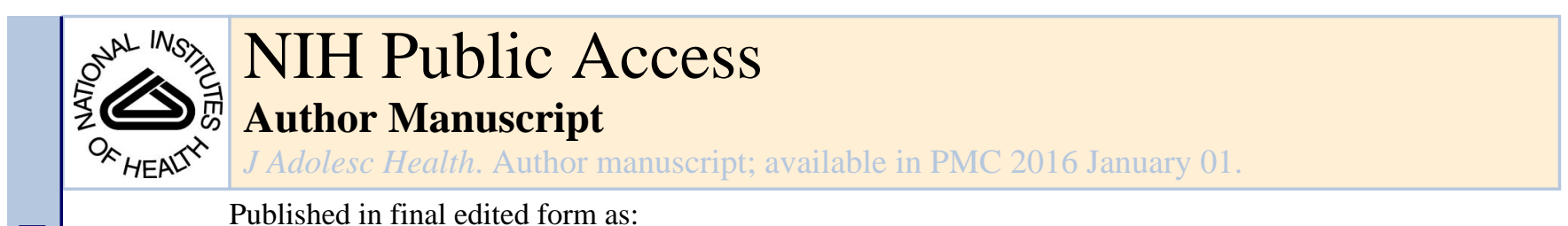

Published in final edited form as:

J Adolesc Health. 2015 January ; 56(1): 66-72. doi:10.1016/j.jadohealth.2014.07.020.

\title{
Associations Between Menarche-related Genetic Variants and Pubertal Growth in Male and Female Adolescents
}

\author{
Wanzhu Tu, Ph.D. ${ }^{a}$, Erin K. Wagner, Ph.D. ${ }^{b}$, George J. Eckert, M.A.S. ${ }^{a}$, Zhangsheng Yu, \\ Ph.D. ${ }^{a}$, Tamara Hannon, M.D.c , J. Howard Pratt, M.D.d,e, and Chunyan He, Sc.D. b,f, \\ aDepartment of Biostatistics, Indiana University School of Medicine, Indianapolis, Indiana \\ bepartment of Epidemiology, Richard M. Fairbanks School of Public Health, Indiana University, \\ Indianapolis, Indiana \\ 'Department of Pediatrics, Indiana University School of Medicine, Indianapolis, Indiana \\ dDepartment of Medicine, Indiana University School of Medicine, Indianapolis, Indiana \\ eThe Veterans Affairs Medical Center, Indianapolis, Indiana \\ ${ }^{f}$ Melvin and Bren Simon Cancer Center, Indiana University, Indianapolis, Indiana
}

\begin{abstract}
Purpose-Previous studies have identified novel genetic variants associated with age at menarche in females of European descent. The pubertal growth effects of these variants have not been carefully evaluated in non-European descent groups. We aimed to examine the effects of 31 newly identified menarche-related single nucleotide polymorphisms (SNPs) on growth outcomes in African American (AA) and European American (EA) children in a prospective cohort.
\end{abstract}

Methods-We analyzed longitudinal data collected from 263 AA and 338 EA enrolled between ages 5 and 17 years; the subjects were followed semiannually for an average of 6 years. The associations between the SNPs and growth-related outcomes, including weight, height, and body mass index (BMI), were examined using mixed-effect models.

Results-Longitudinal analyses revealed that 4 (near or in genes VGLL3, PEX2, CA10, and SKOR2) out of the 14 menarche-only related SNPs were associated with changes in weight and $\mathrm{BMI}$ in EA and AA (P $₫$.0032), but none of them were associated with changes in height. Of the 8 menarche-timing and BMI-related SNPs, none was associated with changes in height, but 3 (in or near genes NEGR1, ETV5, and FTO) were associated with more rapid increases in weight and/or BMI in EA (P 3 .0059). Among the 9 menarche-timing and height-related SNPs, 4 (in or near

(C) 2014 Society of Adolescent Health and Medicine. All rights reserved.

*Address correspondence to: Chunyan He, Sc.D., Department of Epidemiology, Richard M. Fairbanks School of Public Health, Indiana University, 980 West Walnut Street, R3-C241, Indianapolis, Indiana 46202. Tel: 317-278-3033; Fax: 317-278-3966; chunhe@iu.edu (C. He).

The authors declare no conflicts of interest.

Publisher's Disclaimer: This is a PDF file of an unedited manuscript that has been accepted for publication. As a service to our customers we are providing this early version of the manuscript. The manuscript will undergo copyediting, typesetting, and review of the resulting proof before it is published in its final citable form. Please note that during the production process errors may be discovered which could affect the content, and all legal disclaimers that apply to the journal pertain. 
genes ZBTB38, LOC728666, TBX2 and CABLES) were associated with changes in weight or height in EA and AA (P $₫ 0.0042)$.

Conclusion-Genetic variants related to age at menarche were found to be associated with various growth parameters in healthy adolescents. The identified associations were often race and sex-specific.

\section{Keywords}

Age at menarche; genetic variants; puberty; growth

\section{Introduction}

Puberty, the period of transition from childhood to adulthood, is characterized by accelerated growth and sexual maturation. The regulation of puberty is complex and partly influenced by individual's genetic background[1]. Genetic variation affecting events associated with puberty may relate to future disease risks. For example, previous studies have linked younger menarcheal age to increased later-in-life risks of obesity, type 2 diabetes, breast and endometrial cancer, and cardiovascular diseases[2-5]. During puberty, timing of menarche is in general well synchronized with somatic change. A higher childhood body mass index (BMI) has been shown to associate with early onset of menarche[6,7], suggesting a direct influence of adiposity on the timing of sexual maturity. The influence, however, may not be unidirectional as data suggest that female puberty is often accompanied by rapid gains in body weight and body fat $[8,9]$. Although the causal direction remains uncertain, data are consistent on the correlation between menarcheal timing and body fat accumulation, as reflected by increased BMI; this correlation points to the possible existence of common regulating mechanisms shared by these two processes. It is thought that a significant portion of the shared genes account for the correlation between increased childhood adiposity and early sexual maturation as indicated by the onset of menarche[10].

Menarche, the occurrence of the first menstrual cycle, is considered a landmark event of female puberty. Genetic factors are thought to account for approximately $50 \%$ of the observed variation in timing of menarche[11, 12]. Previous genome-wide association studies (GWAS) identified variants in $L I N 28 B$ that associated with age at menarche in women of European ancestry[13-16]. The growth effects of genetic variants in LIN28B have been investigated in three studies in the European and EA populations [14, 17, 18]. However, the growth effects of these genetic compositions in non-white populations are less studied. African American (AA) women are known to have menarche earlier than white women[19, 20], and AA children of both sexes tend to have earlier pubertal growth spurts than children of European ancestry[21]. In addition, genetic variants in LIN28B have also been found to associate with the development of male sexual characteristics (pubic hair and voice change) and pubertal growth in boys[14, 17, 18], suggesting shared genetic mechanisms in boys and girls. It is therefore logical to question whether menarche-related genetic variations relate to growth outcomes similarly in boys and girls as well as in blacks and whites. 
The goal of this study is to explore the genetic influences on pubertal growth. Specifically, we investigated the growth effects of menarche-related genetic variation by using 31 single nucleotide polymorphisms (SNPs) newly identified in an expanded meta-analysis of GWAS of age at menarche among women of European ancestry[22]. Using prospectively collected data from a cohort of AA and EA children and adolescents[23], we evaluated the genetic effects of 31 menarche-related SNPs on various growth measures, including height, weight, and BMI. By assessing the growth effects of these SNPs, we attempted to determine whether there were shared genetic influences underlying menarcheal age and pubertal growth characteristics. Despite the moderate sample size, the frequently repeated measurements and rich phenotypes have provided a unique opportunity to disseminate the growth effects of these SNPs.

\section{Methods}

\section{Study design and subjects}

The study subjects were participants in a longstanding prospective cohort study that was established in 1986 to investigate blood pressure development in children and adolescents. The study design and data collection process have been described elsewhere[23]. Briefly, healthy children between ages 5 and 17 years were recruited from schools in Indianapolis, Indiana, and were followed at 6 month intervals prospectively. The cohort included 601 children, 338 European Americans (EA) and 263 African Americans (AA), who had contributed blood samples for genetic analysis. Data on growth-related outcomes were assessed during the course of follow-up. The study was approved by a local Institutional Review Board. Study subjects or their parents provided informed consent or assent as appropriate.

\section{Outcome assessment}

Height and weight were measured every six months during follow-up[24]. BMI, defined as weight (kilograms)/height (meters) ${ }^{2}$, was calculated for each follow-up visit. On average, each subject contributed 11 repeated measurements. Information on race was ascertained based on the subject's self-report.

\section{SNP selection and genotyping}

We selected 31 independent SNPs that have been reported to be associated with age at menarche in a recent meta-analysis of GWAS of women of European ancestry[11]. Those SNPs were either strongly associated with age at menarche (with reported p-value $\leq$ $1 \times 10^{-8}$ ), or associated with both age at menarche and another adult growth outcome (weight or height) from this report. Based on their associations with age at menarche and other correlated growth traits, we further categorized these SNPs into three groups: Group I included 14 SNPs associated with age at menarche only; Group II included 8 SNPs associated with both age at menarche and adult BMI, and Group III included 9 SNPs associated with both age at menarche and adult height. Detailed descriptions of the SNPs are listed in Table 1. 
DNA was extracted for all participants with blood sample available. Genotypes of the candidate SNPs were determined using the Sequenom MassArray iPLEX Platform (Sequenom, San Diego, CA). The genotyping success rate for each SNP was over 95\%. Samples with percentage of missing genotypes higher than $2 \%$ were removed from the study. In the retained samples, all SNPs were in Hardy-Weinberg equilibrium (P value > 0.05). The allele frequency for each SNP in our data was consistent with that reported for populations of European and African descent in the International HapMap Project (http:// hapmap.ncbi.nlm.nih.gov/).

\section{Statistical analysis}

Weight and height data spanned between ages 5 and 21 years were used in the analyses. Longitudinal analyses were performed using mixed-effect models, which incorporated genetic effects on growth outcomes as fixed effects at the population level; and subjectspecific random effects were incorporated into the model to accommodate the potential correlations among measures from the same subject. The association between each SNP and each measure of growth outcome (weight, height, and BMI) was assessed with adjustment for age, race, and sex, assuming a linear age effect on those outcomes. The models included the main effects of SNP, age, and the SNP*age interactions. The main effect of SNP represented the intercept of the regression line corresponding to the genotype (shift); the age effect represented the rate of growth of the reference genotype (slope); and the SNP*age interaction tested whether the growth rate differed by genotype. Because the true underlying genetic model was unknown, we tested dominant, recessive, and additive genetic models. We then tested three-way interactions SNP*age*race in data after adjusting for the effect of sex, and SNP*age*sex in data after adjusting for the effect of race. These interaction terms allowed us to determine the heterogeneity of the genotype effect on growth rate between males and females, and between EA and AA. In order to control for multiple testing, a Bonferroni correction was used based on the number of SNPs in each group. A statistical significance threshold of $0.0036,0.0063$, and 0.0056 was applied to Group I, II and III, respectively. All P values were based on two-sided tests. Genetic associations and interactions remained significant after adjustment were reported. We additionally explored the genetic effects on various growth outcomes using semiparametric and parametric polynomial models to accommodate the potentially nonlinear growth effects. Graphical exploration did not show strong enough curvature and the results remain similar to those from linear model analysis. We therefore chose to present linear model analysis for convenience of inference. All statistical analyses were performed using SAS version 9.2 software (SAS Institute Inc., Cary, NC, USA).

\section{Results}

\section{Characteristics of subjects}

Participants consisted of 338 EA (170 boys) and 263 AA ( 112 boys) whose characteristics at the time of enrollment and during the follow-up period are presented in Table 2. At the time of study entry, the average age, weight, height, and BMI did not differ significantly between males and females for each of EA and AA groups. However, the average age at study entry 
of EA was younger than that of $\mathrm{AA}(\mathrm{P}=0.0001)$ and $\mathrm{AA}$ were on average heavier, taller, and had a higher BMI compared to EA (all $\mathrm{P}=0.0001$ ).

For the follow-up period, the means of characteristics were obtained by calculating the average of the measurements for the follow-up period, incorporating the repeated measurements on an individual in the standard errors (Table 2). For either EA or AA groups, the mean age and BMI did not differ significantly between males and females. However, males were significantly taller than females $(\mathrm{P}<0.0001)$ and heavier than females in the EA group ( $\mathrm{P}=0.01)$. EA children on average had more follow-up visits than AA children $(\mathrm{P}=0.0001)$. During the study period, AA children were of greater weight, height, and BMI than EA children (all $\mathrm{P}<0.028$ ).

\section{Genetic associations with BMI and weight}

In this longitudinal analysis, we observed associations between several SNPs and changes in weight and/or BMI during the follow-up period. Associations remained significant after adjusting for multiplicity were presented in Table 3 . We expected that SNPs associated with early menarche were associated with either an increased BMI/weight or a more rapid increase in BMI/weight. Of the 14 SNPs with reported association with age at menarche only (group I), rs7642134 near VGLL3 was associated with both an increased weight and BMI in EA females (P $₫ .0016$ ), and the associations were in expected direction as predicted by the SNP association with age at menarche. Two other SNPs, rs7821178 near PEX2 and rs9635759 near $C A 10$, were associated with more rapid gain in BMI and weight in EA (P 4.0032 ), respectively; and rs 1398217 in SKOR2 was associated with more rapid weight gain in AA males $(\mathrm{P}=0.0022)$. However, the three latter associations were not in direction predicted by individual SNP associations with age at menarche.

Of the 8 SNPs with reported association with both age at menarche and adult BMI (group II), we found 3 SNPs that were significantly associated with changes in weight and/or BMI in EA only (Table 3). Two SNPs, rs2815752 near NEGRI and rs7647305 near ETV5, were associated with more rapid gain in both weight and BMI (P $₫$ 0.0042); and rs9939609 in FTO was associated with an increased BMI only $(\mathrm{P}=0.0059)$. The directions of those associations were consistent with individual SNP associations with age at menarche.

Of the 9 SNPs with reported associations with both age at menarche and adult height (group III), two were found to be significantly associated with weight change in AA only (Table 3). Consistent with its association with age at menarche, rs6440003 in ZBTB38 was associated with a rapid weight gain (P $\unlhd .0009$ ); whereas rs757608 near $T B X 2$ was also associated with a rapid weight gain $(\mathrm{P}=0.00003)$, but this association was not in direction predicted by the SNP association with age at menarche.

\section{Genetic associations with height}

None of the 14 menarche-related SNPs (group I) or the 8 menarche and BMI-associated SNPs (group II) were associated with changes in height in our longitudinal analyses.

However, of the 9 SNPs with reported associations with both age at menarche and adult height (group III), three were found to be associated with changes in height in our data 
(Table 3). We expected that SNPs associated with early menarche were associated with either a taller height or a more rapid increase in height during childhood and early adolescence. While rs4549631 near LOC728666 was associated with more rapid gain in height in AA males ( $\mathrm{P}=0.0011)$, rs757608 near $T B X 2$ was associated with more rapid gain in height in AA males and EA (P $₫$.0042). None of these associations were in direction predicted by individual SNP associations with age at menarche. Another SNP, rs4800148 in CABLES1, was associated with increased height in EA males ( $\mathrm{P}=0.0018)$, and this association was in expected direction based on the SNP association with age at menarche.

\section{Race and sex differences}

The associations between candidate SNPs and growth-related outcomes appeared to be raceand sex-specific (Table 3). The majority of the associations were observed in the EA group and only four SNPs were associated with growth outcomes in the AA population. Out of the 11 SNPs associated with growth outcomes in our study, the tests for race differences were significant for 6 SNPs $(\mathrm{P}<0.05)$. On the other hand, approximately $30 \%$ of the associations were observed in males only, predominantly in AA males, and the tests of sex difference were significant for 4 SNPs $(\mathrm{P}<0.05)$.

\section{Discussion}

In this study, we examined the growth effects of menarche-related genetic variations in EA and AA children and adolescents. We found that 11 of the 31 menarche-related SNPs were associated with different aspects of pubertal growth, suggesting shared genetic effects between age at menarche and pubertal growth and pleiotropic effects of these genetic variants. Interestingly, most of the associations were found in relation to the rates of change in growth, which are consistent with evidence suggesting that the rate of growth and weight gain during early childhood may be more critically related to the timing of puberty in boys and girls[26, 27]. In some instances, the identified associations were race- and sexdependent; the fact that we were able to find significant associations after multiplicity adjustment in a relatively small sample, in our opinion, gives more credence to the existence of these influences. The findings point to the existence of a complex series of genetic influences that operate during this time period of rapid growth and development.

The existence of shared mechanisms underlying somatic growth and pubertal development is also supported by a recent GWAS meta-analysis among 18,737 subjects of European ancestry, in which ten genetic loci were found to be associated with pubertal height and growth rates; five of them were also associated with puberty timing[28].The five loci are in or near genes PEX2, VGLL3, LIN28B, MAPK3, and ADCY3. The current study assessed genetic variants at three of these loci (PEX2, VGLL3 and LIN28B). Our data showed that genetic variants at $P E X 2$ and $V G L L 3$ were associated with similar aspects of growth in males and females.

Only three studies have examined the associations between genetic variants at $L I N 28 B$ and growth-related outcomes in populations of European ancestry[14, 17, 18]. In this study, we included one SNP at LIN28B, rs7759938. The associations between rs7759938 and changes in weight, height, and BMI during the follow-up period were not statistically significant, but 
the direction of the associations was consistent with previous studies. This is likely due to our relatively smaller sample size. Additionally, we examined the growth effects of 30 novel menarche-related SNPs for the first time.

Out of the 14 SNPs with previously reported associations with menarche only, we found only 4 (near or in genes VGLL3, PEX2, CA10, and SKOR2) associated with changes in weight and BMI. In addition, for three SNPs the direction of their associations with growth outcomes was unexpected, suggesting the possible existence of different regulatory mechanisms despite common genetic pathways. The paradoxical effects on puberty and somatic growth of these variants suggest the possible involvement of multiple mechanisms that need further investigation[29].

Of the 8 SNPs reported to correlate with earlier menarche and greater adult BMI, we found three (in or near genes NEGR1, ETV5, and FTO) that were associated with faster increases in weight and/or BMI in adolescence. The finding lends support to the notion that common genetic pathways underlie the tracking of childhood and adulthood BMI [30, 31]. Of the 9 SNPs previously associated with menarche and adult height, 4 (in or near genes ZBTB38, $L O C 728666, T B X 2$ and $C A B L E S$ ) were associated with changes in weight or height; two SNPs had effects that were in unexpected directions. Although early puberty is related to shorter adult height in both males and females[32, 33], genetic variants for menarche can exert distinctive, sex-specific effects on pubertal growth as suggested by Widen et al.[18]. Interestingly, we observed these genetic effects on height predominantly in males, consistent with previous findings.

Early puberty in males and females is similarly associated with a faster weight gain and accelerated growth in infancy and early childhood[34], However, marked sex differences in body composition are reported, possibly due to the rise of sex hormones in puberty[35]. Females that are younger at menarche have a higher fat mass over fat-free mass that coincides with changes in estrogen levels[36], whereas males who experience earlier puberty have a higher fat-free mass likely due to changes in androgen levels [37]. It is therefore conceivable that early puberty has different effects on adiposity in males and females. Although it remains largely unknown whether genetic variants influencing the timing of puberty in males and females are the same, one study found a genetic variant at LIN28B, rs314276, was associated with early puberty in both sexes[14]. For females age at menarche and breast development were typically used to determine puberty whereas voice change and pubic hair development were used as indicators for puberty in males[14]. The growth effects of rs314276 and its surrogate (rs7759938) have been investigated in three studies $[14,17,18]$. The findings of those studies are consistent and supportive of the notion that the SNPs are associated with faster height growth during childhood and early adolescence in both sexes, but only in females are they associated with an increased weight and BMI. However, another partially correlated variant at LIN28B, rs314277, has distinctive sex-specific effects on height growth[18]. In our study, we also observed sex-specific genetic effects of menarche-related variants on different aspects of growth outcomes. The complex interactions between genetic variants and sex may partially explain the apparent difference between males and females in early weight gain, insulin sensitivity and hormone changes in relation to puberty development and subsequent growth outcomes[38]. 
In the current study, we investigated 31 candidate SNPs with previously reported associations with age at menarche in EA women[22]. It remains unclear whether genetic variants associated with age at menarche in AA women are similar or different. A recent GWAS of age at menarche has been performed in AA women[25]. Although no SNPs passed the pre-specified threshold for genome-wide significance $\left(\mathrm{P}<5 \times 10^{8}\right)$, this study demonstrated that approximately $60 \%$ of previously identified menarche loci in EA women were associated with menarche in AA women with a relaxed threshold[25], suggesting the cross-ethnic generalization of menarche loci. Specifically, 15 out of the 31 candidate SNPs in our study were found to be associated with age at menarche in both EA and AA women from these reports (Table 1)[22, 25].

What this research does not address is the precise mechanisms by which identified SNPs affect the onset of menarche and pubertal growth. The classical work of Grumbach depicts puberty as a complex neuroendocrine process, one that marked by the reactivation of the hypothalamic-pituitary-gonadal axis, but also influenced by many external factors[39, 40]. The neuroendocrine, genetic, and environmental triggers, as well as their interactions, involved in the control of age at menarche and onset of pubertal growth remain poorly understood. This research highlights the need, and to some extent, the potential target of future investigation on the biological functions of these genetic variants.

The study has a number of important strengths. The study was based on prospective growth assessments with frequent measurements starting in childhood and repeated well into adolescence. The frequently repeated measurements allow us to better quantify the characteristics of growth, and to explore genetic associations with longitudinal changes in somatic growth, including rates of change of growth outcomes. The inclusion of AA subjects in the study cohort has provided valuable data that were unavailable in previous studies.

A number of limitations should be acknowledged. First, the study focused on pubertal growth outcomes instead of pubertal staging. Age at menarche, as well as changes in secondary sexual characteristics such as breast and pubic and facial hair development, and voice change were not collected in the original study. Second, the subjects were children of school age and no early-in-life growth characteristics were available. It is possible that puberty timing-related variants may have important and different growth effects during this period, as rapid weight gain in infancy and a faster growth rate within the first 2 years of life are critical and have been linked to an earlier puberty[7,34] and body composition in adolescents[38]. Finally, the study power was limited due to the relatively small sample size. As a result, genetic associations of moderate effect sizes may not be detected. The analytic power was more limited in race and sex-specific subgroup analyses. As in all studies, lack of statistical significance could be due to limited power, thus should not be interpreted as evidence for lack of association. Further confirmatory studies should be conducted to validate the reported findings.

In conclusion, genetic variants associated with menarche timing are found to correlate with somatic growth and weight change in adolescents, suggesting shared genetic factors underlying age at menarche and pubertal growth. These reported associations appear in 
some instances to be sex- and race- specific. The findings require replication in other populations and large studies. Future studies may identify relationships between these genetic variations and the risk for disorders that occur post puberty.

\section{Acknowledgments}

The authors thank Drs. Howard J. Edenberg and Xiaoling Xuei, and the technical staff at the Center for Medical Genomics, Indiana University School of Medicine for their collaboration and scientific support in performing the genotyping for the study. This research is supported by an Indiana CTSI grant, the NIH grant RO1 HL095086 and a VA Merit Review grant. The authors also thank Regenstrief Institute Center for Biomedical Informatics for their support of this research.

\section{References}

1. Gajdos ZK, Henderson KD, Hirschhorn JN, et al. Genetic determinants of pubertal timing in the general population. Mol Cell Endocrinol. 2010; 324:21-9. [PubMed: 20144687]

2. Kvale G. Reproductive factors in breast cancer epidemiology. Acta Oncol. 1992; 31:187-94. [PubMed: 1622633]

3. Purdie DM, Green AC. Epidemiology of endometrial cancer. Best Pract Res Clin Obstet Gynaecol. 2001; 15:341-54. [PubMed: 11476557]

4. He C, Zhang C, Hunter DJ, et al. Age at menarche and risk of type 2 diabetes: results from 2 large prospective cohort studies. Am J Epidemiol. 2010; 171:334-44. [PubMed: 20026580]

5. Lakshman R, Forouhi NG, Sharp SJ, et al. Early age at menarche associated with cardiovascular disease and mortality. J Clin Endocrinol Metab. 2009; 94:4953-60. [PubMed: 19880785]

6. Freedman DS, Khan LK, Serdula MK, et al. The relation of menarcheal age to obesity in childhood and adulthood: the Bogalusa heart study. BMC Pediatr. 2003; 3:3. [PubMed: 12723990]

7. Ong KK, Emmett P, Northstone K, et al. Infancy weight gain predicts childhood body fat and age at menarche in girls. J Clin Endocrinol Metab. 2009; 94:1527-32. [PubMed: 19240149]

8. Demerath EW, Li J, Sun SS, et al. Fifty-year trends in serial body mass index during adolescence in girls: the Fels Longitudinal Study. Am J Clin Nutr. 2004; 80:441-6. [PubMed: 15277168]

9. Jasik CB, Lustig RH. Adolescent obesity and puberty: the "perfect storm". Ann N Y Acad Sci. 2008; 1135:265-79. [PubMed: 18574233]

10. Kaprio J, Rimpela A, Winter T, et al. Common genetic influences on BMI and age at menarche. Hum Biol. 1995; 67:739-53. [PubMed: 8543288]

11. Sharma K. Genetic basis of human female pelvic morphology: a twin study. Am J Phys Anthropol. 2002; 117:327-33. [PubMed: 11920368]

12. Towne B, Czerwinski SA, Demerath EW, et al. Heritability of age at menarche in girls from the Fels Longitudinal Study. Am J Phys Anthropol. 2005; 128:210-9. [PubMed: 15779076]

13. He C, Kraft P, Chen C, et al. Genome-wide association studies identify loci associated with age at menarche and age at natural menopause. Nat Genet. 2009; 41:724-8. [PubMed: 19448621]

14. Ong KK, Elks CE, Li S, et al. Genetic variation in LIN28B is associated with the timing of puberty. Nat Genet. 2009; 41:729-33. [PubMed: 19448623]

15. Perry JR, Stolk L, Franceschini N, et al. Meta-analysis of genome-wide association data identifies two loci influencing age at menarche. Nat Genet. 2009; 41:648-50. [PubMed: 19448620]

16. Sulem P, Gudbjartsson DF, Rafnar T, et al. Genome-wide association study identifies sequence variants on 6q21 associated with age at menarche. Nat Genet. 2009; 41:734-8. [PubMed: 19448622]

17. Ong KK, Elks CE, Wills AK, et al. Associations between the pubertal timing-related variant in LIN28B and BMI vary across the life course. J Clin Endocrinol Metab. 2011; 96:E125-9. [PubMed: 20962026]

18. Widen E, Ripatti S, Cousminer DL, et al. Distinct variants at LIN28B influence growth in height from birth to adulthood. Am J Hum Genet. 2010; 86:773-82. [PubMed: 20398887] 
19. Freedman DS, Khan LK, Serdula MK, et al. Relation of age at menarche to race, time period, and anthropometric dimensions: the Bogalusa Heart Study. Pediatrics. 2002; 110:e43. [PubMed: 12359816]

20. Wu T, Mendola P, Buck GM. Ethnic differences in the presence of secondary sex characteristics and menarche among US girls: the Third National Health and Nutrition Examination Survey, 1988-1994. Pediatrics. 2002; 110:752-7. [PubMed: 12359790]

21. Tu W, Eckert GJ, Saha C, et al. Synchronization of adolescent blood pressure and pubertal somatic growth. J Clin Endocrinol Metab. 2009; 94:5019-22. [PubMed: 19850686]

22. Elks CE, Perry JR, Sulem P, et al. Thirty new loci for age at menarche identified by a metaanalysis of genome-wide association studies. Nat Genet. 2010; 42:1077-85. [PubMed: 21102462]

23. Pratt JH, Jones JJ, Miller JZ, et al. Racial differences in aldosterone excretion and plasma aldosterone concentrations in children. N Engl J Med. 1989; 321:1152-7. [PubMed: 2677724]

24. Tu W, Eckert GJ, DiMeglio LA, et al. Intensified effect of adiposity on blood pressure in overweight and obese children. Hypertension. 2011; 58:818-24. [PubMed: 21968752]

25. Demerath EW, Liu CT, Franceschini N, et al. Genome-wide association study of age at menarche in African-American women. Hum Mol Genet. 2013; 22:3329-46. [PubMed: 23599027]

26. Cooper C, Kuh D, Egger P, et al. Childhood growth and age at menarche. Br J Obstet Gynaecol. 1996; 103:814-7. [PubMed: 8760713]

27. Mills JL, Shiono PH, Shapiro LR, et al. Early growth predicts timing of puberty in boys: results of a 14-year nutrition and growth study. J Pediatr. 1986; 109:543-7. [PubMed: 3746550]

28. Cousminer DL, Berry DJ, Timpson NJ, et al. Genome-wide association and longitudinal analyses reveal genetic loci linking pubertal height growth, pubertal timing and childhood adiposity. Hum Mol Genet. 2013; 22:2735-47. [PubMed: 23449627]

29. Ahmed ML, Ong KK, Dunger DB. Childhood obesity and the timing of puberty. Trends Endocrinol Metab. 2009; 20:237-42. [PubMed: 19541497]

30. Guo SS, Wu W, Chumlea WC, et al. Predicting overweight and obesity in adulthood from body mass index values in childhood and adolescence. Am J Clin Nutr. 2002; 76:653-8. [PubMed: 12198014]

31. Monteiro PO, Victora CG. Rapid growth in infancy and childhood and obesity in later life--a systematic review. Obes Rev. 2005; 6:143-54. [PubMed: 15836465]

32. Carel JC, Lahlou N, Roger M, et al. Precocious puberty and statural growth. Hum Reprod Update. 2004; 10:135-47. [PubMed: 15073143]

33. Onland-Moret NC, Peeters PH, van Gils CH, et al. Age at menarche in relation to adult height: the EPIC study. Am J Epidemiol. 2005; 162:623-32. [PubMed: 16107566]

34. Dunger DB, Ahmed ML, Ong KK. Early and late weight gain and the timing of puberty. Mol Cell Endocrinol. 2006; 254-255:140-5.

35. Ahmed ML, Ong KK, Morrell DJ, et al. Longitudinal study of leptin concentrations during puberty: sex differences and relationship to changes in body composition. J Clin Endocrinol Metab. 1999; 84:899-905. [PubMed: 10084568]

36. Bandini LG, Must A, Naumova EN, et al. Change in leptin, body composition and other hormones around menarche--a visual representation. Acta Paediatr. 2008; 97:1454-9. [PubMed: 18657126]

37. Rogol AD. Growth at puberty: interaction of androgens and growth hormone. Med Sci Sports Exerc. 1994; 26:767-70. [PubMed: 7914344]

38. Botton J, Heude B, Maccario J, et al. Postnatal weight and height growth velocities at different ages between birth and $5 \mathrm{y}$ and body composition in adolescent boys and girls. Am J Clin Nutr. 2008; 87:1760-8. [PubMed: 18541566]

39. Grumbach MM. The neuroendocrinology of human puberty revisited. Horm Res. 2002; 57 (Suppl 2):2-14. [PubMed: 12065920]

40. Roth CL, Ojeda SR. Genes involved in the neuroendocrine control of normal puberty and abnormal puberty of central origin. Pediatr Endocrinol Rev. 2005; 3:67-76. [PubMed: 16361980] 


\section{Implications and Contribution}

This study examines the growth effects of menarche-related variants in individuals of European and non-European ancestries. The findings indicate that genetic variants responsible for age at menarche are associated with pubertal growth in healthy adolescents. Data suggest shared genetic influences on menarcheal age and pubertal growth. 


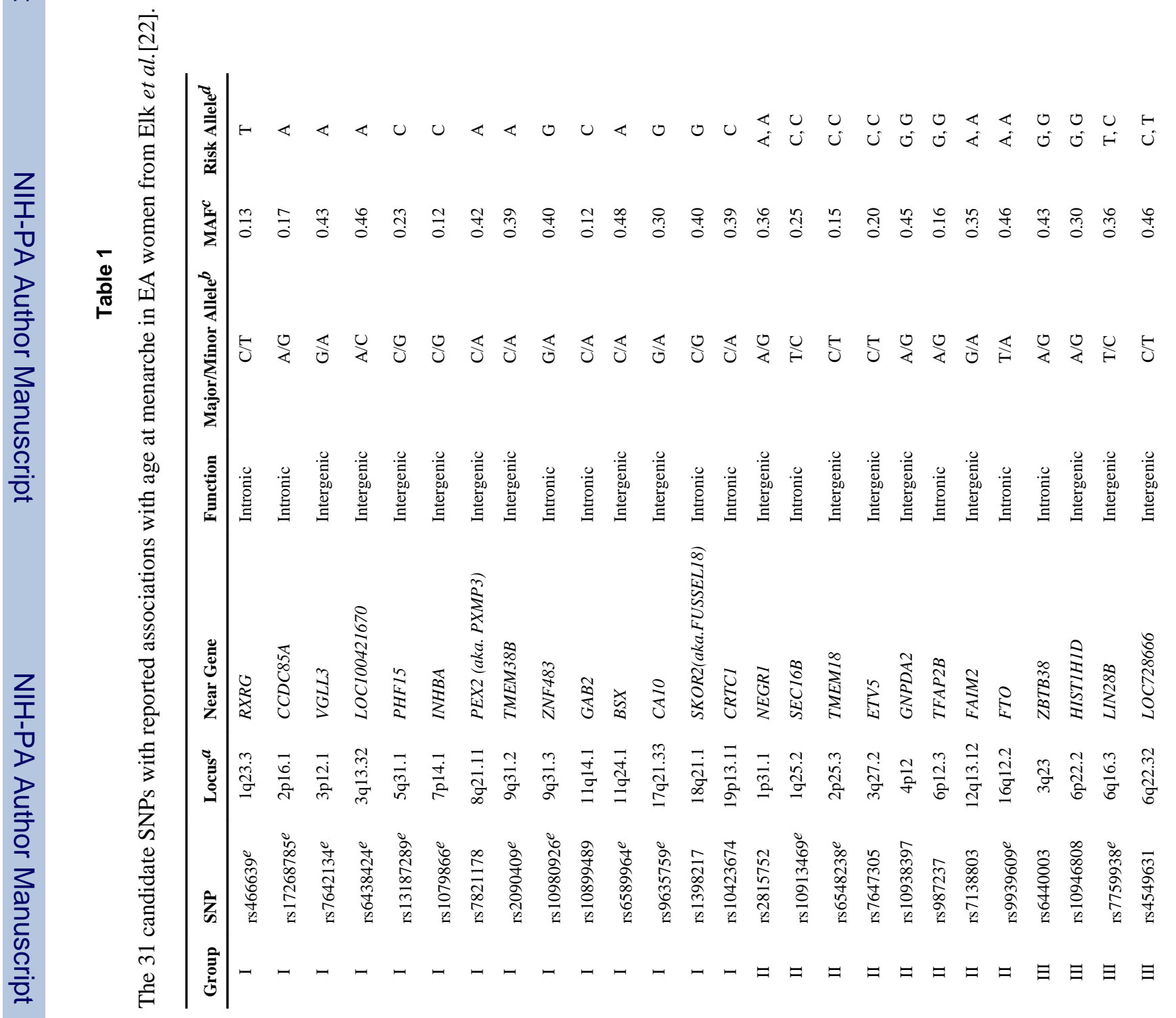




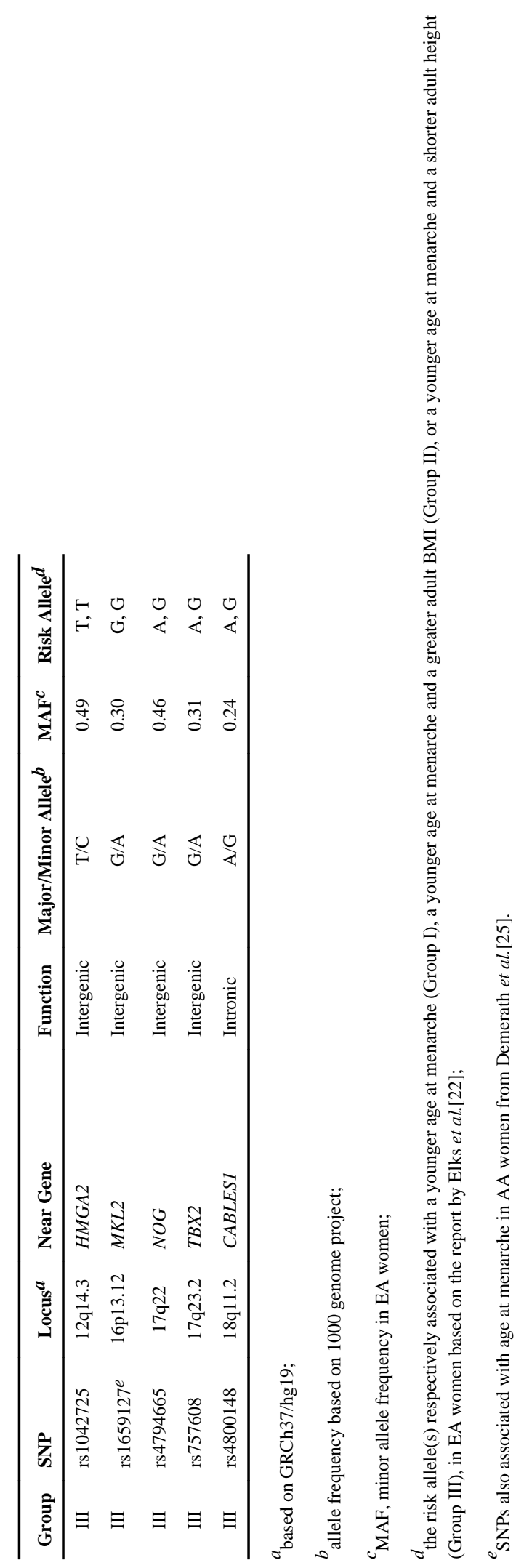




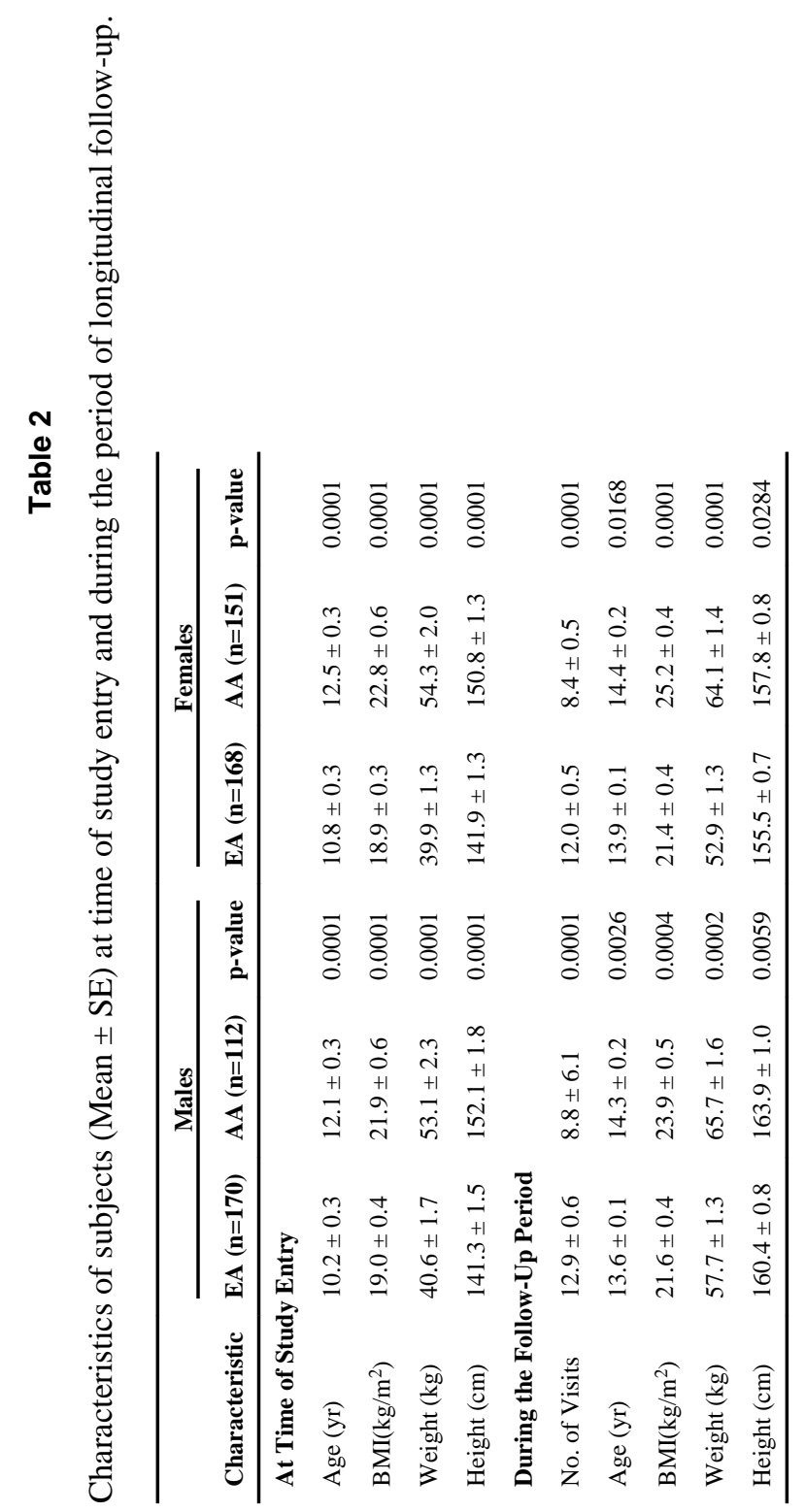




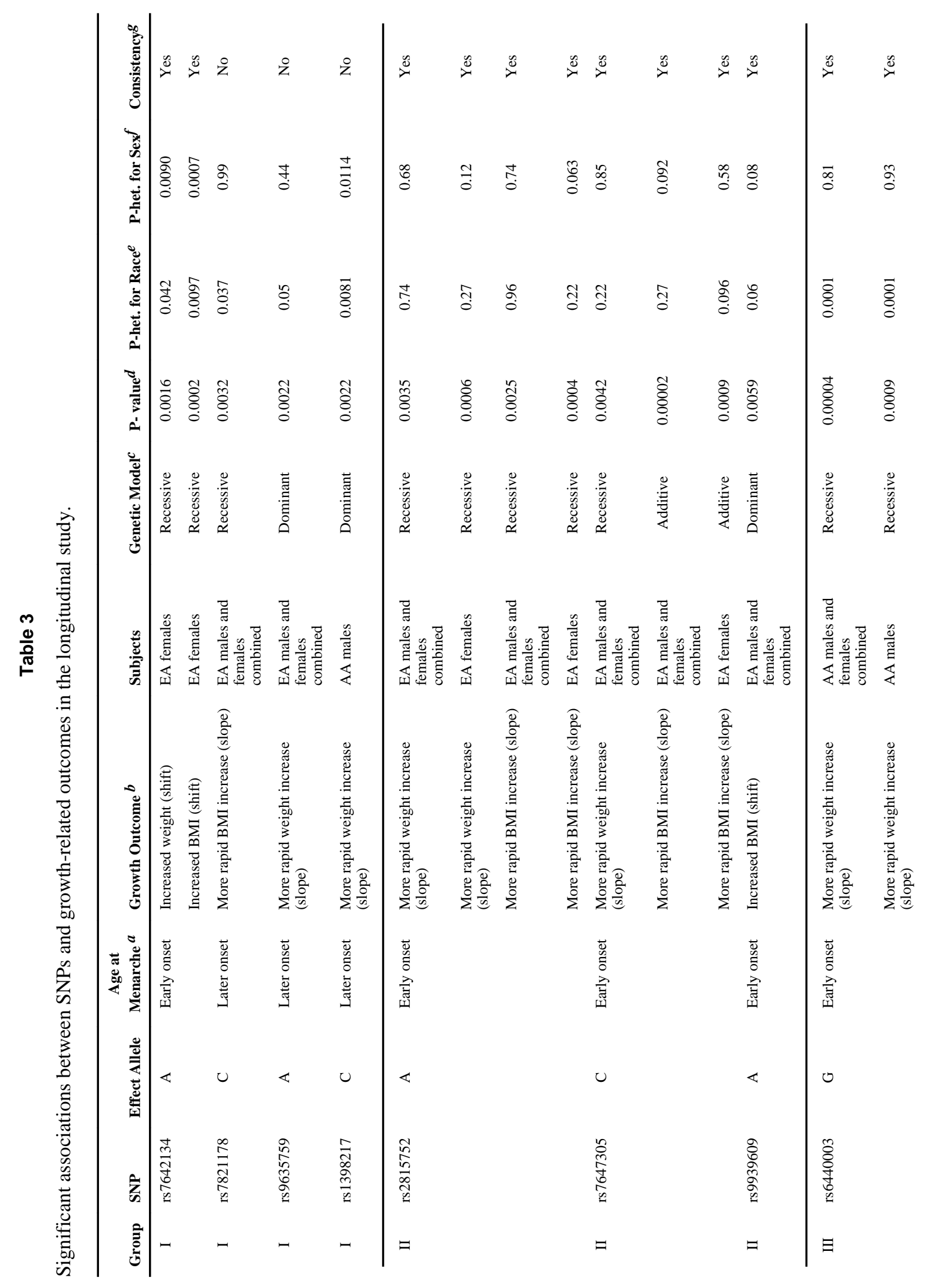




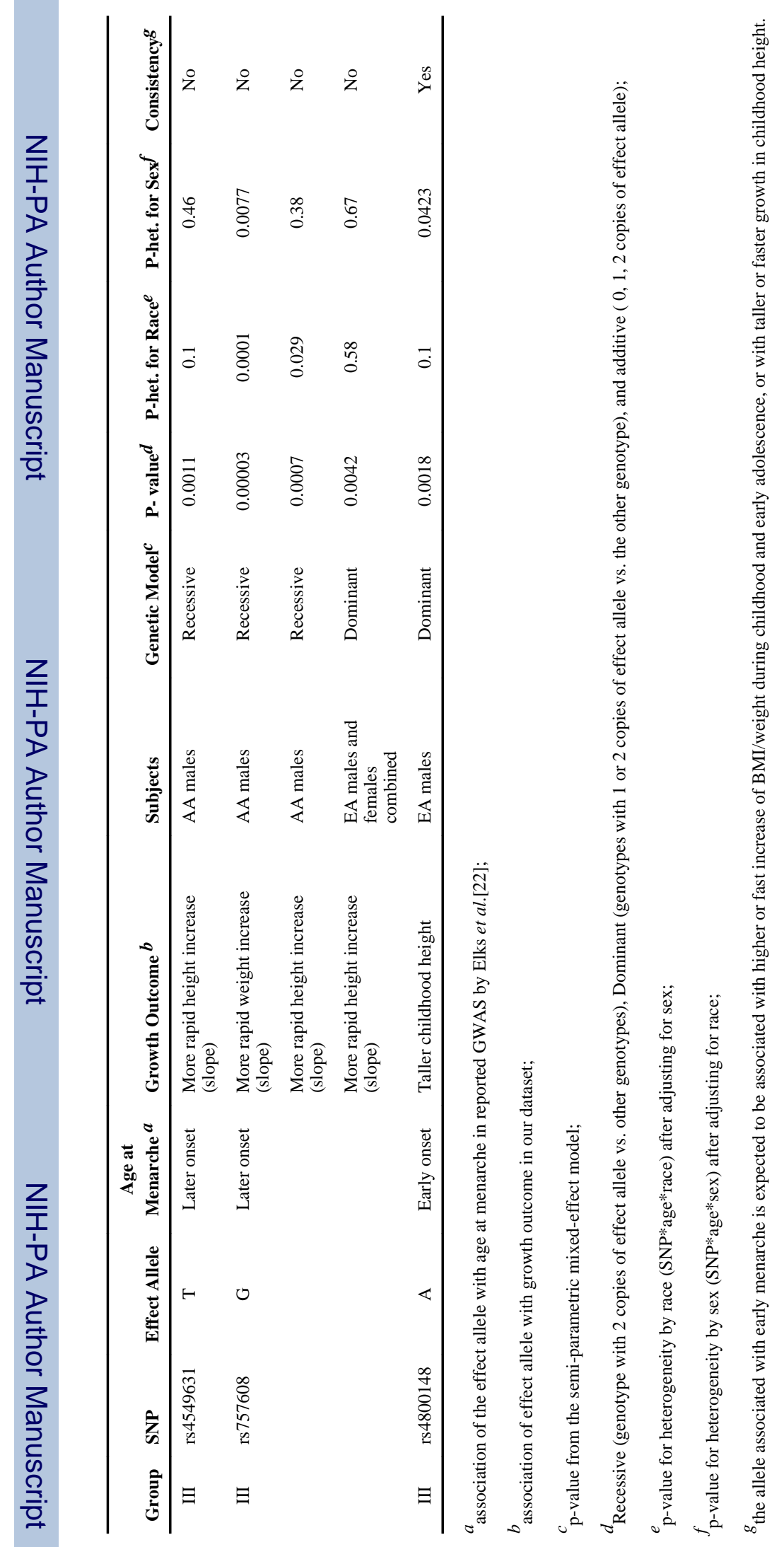

$J$ Adolesc Health. Author manuscript; available in PMC 2016 January 01. 